\title{
Prevalence of Bisphosphonate-related Osteonecrosis of the Jaw-like Lesions Is Increased in a Chemotherapeutic Dose-dependent Manner in Mice
}

Shinichiro Kuroshima a,b, ", Muneteru Sasaki a , Kazunori Nakajima a, Saki Tamaki a, Hiroki Hayano a, Takashi Sawase ${ }^{a}$

${ }^{a}$ Department of Applied Prosthodontics, Graduate School of Biomedical Sciences, Nagasaki University, 1-7-1, Sakamoto, Nagasaki, 852-8588, Japan

b Oral \& Maxillofacial Implant Center, Nagasaki University Hospital, 1-7-1, Sakamoto, Nagasaki, 8528588, Japan

"Corresponding author

E-mail address: kuroshima@nagasaki-u.ac.jp (S. Kuroshima)

S Kuroshima and M Sasaki equally contributed to this work.

\section{Highlights}

- Impaired tooth socket healing rarely occurred with cyclophosphamide (CY) monotherapy.

- Bisphosphonate (ZA) monotherapy did not induce BRONJ-like lesions in mice.

- High-dose CY in combination with ZA and tooth extraction induced BRONJ-like lesions in mice.

- BRONJ-like lesions prevalently increased in a CY dose-dependent manner. 


\section{Abstract}

Bisphosphonate-related osteonecrosis of the jaw (BRONJ) worsens oral health-related quality of life. Most BRONJ occurs in multiple myeloma or metastatic breast cancer patients treated with bisphosphonate/chemotherapeutic combination therapies. Cyclophosphamide (CY), an alkylating chemotherapeutic drug, is used to treat multiple myeloma, although its use has been recently reduced. The aim of this study was to clarify the effects of CY dose on tooth extraction socket healing when CY is used with or without bisphosphonate in mice. Low-dose CY (50 mg/kg; CY-L), moderate-dose CY (100 mg/kg; CY-M), high-dose CY (150 mg/kg; CY-H), and bisphosphonate [Zometa (ZA): $0.05 \mathrm{mg} / \mathrm{kg}$ ] were administered for 7 weeks. Each dose of CY and ZA in combination was also administered for 7 weeks. Both maxillary first molars were extracted at 3 weeks after the initiation of drug administration. Euthanasia was performed at 4 weeks post-extraction. Gross wound healing, microcomputed tomography analysis, histomorphometry, and immunohistochemistry were used to qualitatively evaluate osseous and soft tissue wound healing of tooth extraction sockets. ZA monotherapy induced no BRONJ-like lesions in mice. CY monotherapy rarely induced open wounds, though delayed osseous wound healing occurred in a CY dose-dependent manner. In contrast, CY/ZA combination therapy prevalently induced BRONJlike lesions with compromised osseous and soft tissue healing in a CY dose-dependent manner. Interestingly, anti-angiogenesis was noted regardless of CY dose and ZA administration, even though only $\mathrm{CY}-\mathrm{M} / \mathrm{ZA}$ and $\mathrm{CY}-\mathrm{H} / \mathrm{ZA}$ combination therapies induced BRONJ-like lesions. Our findings suggest that high-dose CY may be associated with the development of BRONJ following tooth extraction only when $C Y$ is used together with ZA. In addition to anti-angiogenesis, other factors may contribute to the pathoetiology of BRONJ.

Keywords: bisphosphonates, cyclophosphamide, bisphosphonate-related osteonecrosis of the jaw, wound healing, dose effect 


\section{Introduction}

Nitrogen-containing bisphosphonates bind and inhibit a key enzyme of the intracellular mevalonate pathway, thereby resulting in apoptosis of osteoclasts. In general, oral bisphosphonates are used to treat osteoporosis, whereas intravenous bisphosphonates are utilized to reduce the risks of skeletal-related events such as pathological vertebral fracture and bone pain in oncology or metastasis patients [1]. However, in 2003, bisphosphonate-related osteonecrosis of the jaw (BRONJ) was first reported to occur following tooth extraction in patients taking intravenous bisphosphonates [2]. BRONJ is defined as "exposed bone or bone that can be probed through an intraoral or extraoral fistula in the maxillofacial region that has persisted for more than 8 weeks" [3]. The prevalence of BRONJ is rare, occurring in $0.05 \%$ of oral bisphosphonate users following tooth extraction, and $1.6 \%-14.8 \%$ of intravenous bisphosphonate users following tooth extraction [4]. A systematic review reported that BRONJ most frequently occurs in multiple myeloma or metastatic breast cancer patients taking intravenous bisphosphonates [5]. Tooth extraction (61.7\%), dental implant treatment (3.9\%), dental surgery $(7.2 \%)$, periodontal disease $(5.0 \%)$, prosthetic trauma such as ill-fitting removable prostheses (7.4\%), and spontaneous cases (14.8\%) are triggering factors of BRONJ [5], although all dental therapies including tooth cleaning, root canal filling, and prosthodontic treatment are not contraindicated in patients taking bisphosphonates. BRONJ worsens oral health-related quality of life [6], which results in negative effects on daily life and social activities. However, the exact mechanism of BRONJ has not been clear. Moreover, definitive treatment strategies for BRONJ have not been developed due to its uncertain mechanisms. Therefore, elucidation of the pathoetiology and pathophysiology of BRONJ is required.

Multiple myeloma, which represents $1 \%$ of all cancers and approximately $10 \%$ of all hematologic malignancies, occurs in 20,000 people in the United States every year [7]. Moreover, the age-adjusted 5-year relative survival rate increased from $39.8 \%$ to $53.2 \%$ [7]. The increase in survival rate of multiple myeloma patients is due to the development of therapeutic strategies. A current review reported that thalidomide, lenalidomide, bortezomib, carfilzomib, pomalidomide, cyclophosphamide, melphalan, dexamethasone, and prednisone are used to treat multiple myeloma solely or adjunctively [8]. 
Generally, cyclophosphamide or melphalan are utilized for the treatment of multiple myeloma in combination with intravenous bisphosphonates that reduce the risks of skeletal complications [9]. The use of cyclophosphamide or melphalan in combination with prednisone, thalidomide, bortezomib, or carfilzomib was introduced as the major treatment regimens for multiple myeloma in 2016 [8], whereas at the 2017 American Society of Hematology Annual Meeting, the use of cyclophosphamide and melphalan were reportedly decreased [10]. Different doses of cyclophosphamide are administered to multiple myeloma patients depending on the patient's age and tolerability [9]. However, the effects of different doses of cyclophosphamide on tooth extraction socket healing, with or without bisphosphonate therapy, are not fully understood.

The aims of the present study were 1) to clarify the effects of cyclophosphamide dose on tooth extraction socket healing, and 2) to investigate the effects of each dose of cyclophosphamide and bisphosphonate combination therapy on osseous and soft tissue wound healing of tooth extraction sockets in C57BL6/J mice.

\section{Materials and methods}

\subsection{Animals, tooth extraction, and drug therapies}

Eight- to twelve-week-old male C57BL/6J mice were used (CLEA Japan Inc., Osaka, Japan). The duration of drug therapies was 7 weeks. Both maxillary first molars were extracted at 3 weeks after the start of drug therapy. All mice were euthanized at 4 weeks post-extraction. Subcutaneous injection of zoledronate (Zometa; Novartis, Stein, Switzerland) was performed at $0.05 \mathrm{mg} / \mathrm{kg}$ twice a week for 7 weeks (ZA, $n=7)$. Intraperitoneal injection of cyclophosphamide (C7397; Sigma-Aldrich, St. Louis, MO, USA) was carried out at $50 \mathrm{mg} / \mathrm{kg}$ (low dose; CY-L, $\mathrm{n}=7$ ) and $100 \mathrm{mg} / \mathrm{kg}$ (moderate dose; CY-M, $\mathrm{n}=7$ ) twice a week for 7 weeks. Additionally, $150 \mathrm{mg} / \mathrm{kg}$ of $\mathrm{CY}$ (high dose; $\mathrm{CY}-\mathrm{H}, \mathrm{n}=7$ ) was intraperitoneally injected twice and once a week before and after tooth extraction for 7 weeks, respectively. ZA was also subcutaneously injected for 7 weeks in combination with CY-L, CY-M, and CY-H (CY-L/ZA, CY-M/ZA, and $\mathrm{CY}-\mathrm{H} / \mathrm{ZA}$, respectively, $\mathrm{n}=7 /$ each group). Saline was used as a control $(\mathrm{VC}, \mathrm{n}=7)($ Fig. 1). Animal 
care and experimental procedures were performed in accordance with the Guidelines for Animal Experimentation of Nagasaki University, with approval from the Ethics Committee for Animal Research.

\subsection{Microcomputed tomography (MicroCT)}

After euthanizing mice, right maxillae including tooth extraction sites were dissected and fixed in $10 \%$ neutral buffered formalin for 24 hours. They were scanned using microCT at $20-\mu \mathrm{m}$ voxel resolution and 90-kV tube voltage (R_mCT2; Rigaku Co. Ltd., Tokyo, Japan) [11]. Segmentation and reconstruction of tooth extraction sockets were performed with a semi-manual contouring method using TRI/3D-Bon (Ratoc System Engineering, Tokyo, Japan) [12]. In tooth extraction sockets, the bone mass of the sockets (bone volume fraction; BVF), trabecular number (Tb.N), trabecular thickness (Tb.Th), trabecular separation (Tb.Sp), and bone mineral density (BMD) were semi-automatically measured in accordance with the guidelines for assessment of bone microstructures using microCT [13].

\subsection{Histomorphometry}

Left maxillae were fixed in $10 \%$ neutral formalin at euthanasia. The maxillae were demineralized in $10 \%$ EDTA, paraffin embedded, and sectioned at $5-\mu \mathrm{m}$ thickness in serial sagittal sections. Tartrateresistant acid phosphatase (TRAP) staining was performed to detect osteoclasts on bone surfaces (386A; Sigma-Aldrich). Masson's trichrome staining was conducted for the visualization of collagen fibers (HT15; Sigma-Aldrich). Hematoxylin and eosin (H-E) staining was carried out for the evaluation of soft and hard tissues. Sustained bone exposure without epithelial coverage for more than 8 weeks is required to diagnose BRONJ in humans [4]. It requires 2 and more than 6 weeks to complete bone remodeling in mice and humans, respectively $[14,15]$. In this study, osseous and soft tissue wounds with impaired healing and constant bone exposure in the oral cavity for 4 weeks were designated as BRONJ-like lesions, since tooth extraction socket healing in mice is shorter than that in humans. Therefore, the validation of BRONJ-like lesions in this study was histomorphometrically performed by reference to previous reports that validated human BRONJ $[16,17]$ as follows: 1) osteoclast numbers on the bone surface per bone 
linear perimeter in tooth extraction sockets (N.Oc/BS, \#/mm); 2) vital bone area in which there were morphologically normal osteocytes in tooth extraction sockets [living bone (\%)]; 3) necrotic bone, defined as the bone area where there are $\geq 10$ adjacent empty or pyknotic osteocyte lacunae that is non-vital [18, 19] [necrotic bone (\%)]; 4) the number of empty osteocyte lacunae in tooth extraction sockets [empty lacunae $\left(\# / \mathrm{mm}^{2}\right)$ ]; 5) osteocyte numbers of tooth extraction sockets [osteocyte density $\left(\# / \mathrm{mm}^{2}\right)$ ]; 6) collagen fibers quantified in the remaining connective tissue [area of interest (AOI), $200 \mu \mathrm{m} \times 500 \mu \mathrm{m}$ ] above the extraction sockets [collagen production (\%)]; and 7) infiltration of polymorphonuclear cells (PMN) assessed by quantifying them in the remaining connective tissue within $100 \mu \mathrm{m}$ of the bone surface $(\mathrm{AOI}, 100 \mu \mathrm{m} \times 500 \mu \mathrm{m})\left(\mathrm{PMN}\right.$ infiltration, $\left.\# / \mathrm{mm}^{2}\right)$. It has been reported that higher magnification of $\mathrm{H}$-E-stained sections can revealed the presence of PMNs [20]. Hence, in this study, PMN infiltration was evaluated with $\mathrm{H}-\mathrm{E}$ stained sections under higher magnification (x400), according to the previous study.

\subsection{Immunohistochemistry}

To detect blood vessels, the sections were incubated with a CD31 rabbit anti-mouse primary antibody (ab56299; Abcam, Cambridge, MA, USA) (1:100 dilution) and fluorescent-conjugated goat antirat Alexa Flour 594 (secondary antibody; Invitrogen, Carlsbad, CA, USA) (1:200 dilution), and mounted using VECTASHIELD Antifade Mounting Medium with DAPI (H-1200 Vector Laboratories, Burlingame, CA, USA). Immune-labeled sections were visualized using immunofluorescent microscopy (Axio Scope A1, Zeiss, Oberkochen, Germany). Blood vessel numbers and areas were quantitatively analyzed by semi-automatically counting the number and area of vessels in the remaining connective tissue above the tooth extraction sockets $(\mathrm{AOI}, 200 \mu \mathrm{m} \times 500 \mu \mathrm{m})$ by reference to our previous study [21].

\subsection{Statistics}

Statistical analyses were blindly conducted. The Shapiro-Wilk test was performed for normality. One-way analysis of variance (ANOVA) for multiple groups was used for parametric data. The Kruskal- 
Wallis test for multiple groups was used for non-parametric data. All statistical analyses were conducted using Systat 12 (Systat Software, Chicago, IL, USA). An a-level of 0.05 was used for statistical significance. All data are represented as means \pm SEM.

\section{Results}

\subsection{Effect of CY monotherapy dose on osseous healing of tooth extraction sockets}

No open wounds were observed in the VC ( 0 out of 14 extraction sockets) and CY-L ( 0 out of 14 extraction sockets) groups at 4 weeks after tooth extraction. However, $7.14 \%$ (1 out of 14 extraction sockets) and $14.2 \%$ (2 out of 14 extraction sockets) open wounds were noted in CY-M and CY-H groups, respectively, at 4 weeks after tooth extraction (Fig. 2a). Hard tissue healing appeared to be the same between VC and CY-L groups (Fig. 2b). CY monotherapy for 7 weeks did not change osteoclast numbers in the tooth extraction sockets irrespective of CY dose (Fig. 2b-C). CY-L monotherapy did not alter living bone, necrotic bone area, empty lacunae, or osteocyte numbers (Fig. 2d-g). Conversely, bone responses

were different between $\mathrm{VC}$ and $\mathrm{CY}$ therapy when the $\mathrm{CY}$ dose was higher. $\mathrm{CY}-\mathrm{M}$ and $\mathrm{CY}-\mathrm{H}$ monotherapies significantly decreased the living bone and osteocyte numbers with increases in necrotic bone and empty lacunae compared with VC or sometimes CY-L monotherapy (Fig. 2b, 2d-g).

\subsection{Effect of $\mathrm{CY}$ monotherapy dose on bone architecture of tooth extraction sockets}

We also investigated the bone architecture of tooth extraction sockets. Bone structures appeared to be the same between VC and CY-L groups (Fig. 3a). Bone fill, Tb.N, Tb.Th, and Tb.Sp were all similar between VC and CY-L (Fig. 3b-e). However, bone architectures were different in CY-M and CY-H groups. CY-M and $\mathrm{CY}-\mathrm{H}$ monotherapies significantly decreased bone fill of tooth extraction sockets when compared with those in VC and CY-L groups (Fig. 3b). CY-H monotherapy also severely decreased bone fill compared with CY-M monotherapy (Fig. 3b). CY-M and CY-H monotherapies significantly decreased Tb.N when compared with those in VC and CY-L groups (Fig. 3c). Tb.Th was nearly the same among all groups regardless of $\mathrm{CY}$ dose (Fig. 3d). CY-H monotherapy significantly increased Tb.Sp when compared 
with all other groups (Fig. 3e). CY monotherapy significantly decreased BMD of tooth extraction sockets compared with VC regardless of CY dose (Fig. 3f).

\subsection{Effect of CY monotherapy dose on soft tissue healing of tooth extraction sockets}

We next investigated the effects of $\mathrm{CY}$ dose on soft tissue healing of tooth extraction sockets. Soft tissue healing seemed to be similar irrespective of CY dose (Fig. 4a), and the production of collagen fibers was almost the same regardless of dose (Fig. 4b). In addition, PMNs had infiltrated irrespective of CY dose (Fig. 4c). We next quantitatively analyzed the effects of $\mathrm{CY}$ dose on angiogenesis in tooth extraction sockets. CY monotherapy significantly suppressed the number and area of blood vessels, irrespective of $\mathrm{CY}$ dose (Fig. 4b, e-f).

\subsection{Effect of CY/ZA combination therapy on osseous healing of tooth extraction sockets}

No open wounds were observed following VC or ZA monotherapy ( 0 out of 14 extraction sockets) or CY-L/ZA combination therapy (0 out of 14 extraction sockets) at sacrifice. However, $21.6 \%$ (3 out of 14 extraction sockets) and $92.8 \%$ (13 out of 14 extraction sockets) open wounds were noted in CY-M/ZA and $\mathrm{CY}-\mathrm{H} / \mathrm{ZA}$ groups, respectively, at 4 weeks after tooth extraction (Fig. 5a-b). ZA administration significantly suppressed osteoclast numbers in tooth extraction sockets irrespective of $\mathrm{CY}$ administration (Fig. 5b-c). However, ZA monotherapy did not change the living bone area compared with VC (Fig. 5d). CY/ZA combination therapies significantly decreased the living bone in sockets compared with VC/ZA monotherapy (Fig. 5d). ZA monotherapy increased necrotic bone without a statistically significant difference, whereas CY/ZA combination therapies significantly increased the necrotic bone area compared with VC and ZA monotherapies regardless of $\mathrm{CY}$ dose (Fig. 5e). The number of empty lacunae was the same between VC and ZA monotherapies; however, CY/ZA combination therapies significantly increased the number of empty lacunae compared with VC and ZA monotherapies (Fig. 5f). Additionally, administration of ZA administration significantly decreased the number of osteocytes in the bone of tooth extraction sockets, irrespective of CY injection (Fig. 5g). 


\subsection{Effect of CY/ZA combination therapy on bone architecture of tooth extraction sockets}

MicroCT scans were carried out to evaluate the bone structures of the sockets. ZA monotherapy significantly increased bone fill of tooth extraction compared with VC without open wounds (Fig. 6a). CYH/ZA combination therapy significantly decreased bone fill of tooth extraction sockets when compared with all other groups (Fig. 6b). ZA monotherapy and CY-M/ZA and CY-H/ZA combination therapies significantly decreased Tb.N compared with VC (Fig. 6c). CY-H/ZA combination therapy significantly decreased Tb. Th and increased Tb.Sp when compared with all other groups (Fig. 6d and 6e, respectively). ZA therapy significantly increased BMD compared with VC. However, CY/ZA combination therapy significantly decreased BMD when compared with VC and ZA monotherapy, irrespective of CY dose (Fig. 6f)

\subsection{Effect of CY/ZA combination therapy on soft tissue healing of tooth extraction sockets}

Finally, we assessed the combinatory effect of $C Y$ and ZA on soft tissue healing of tooth extraction sockets. CY-H/ZA significantly decreased collagen production and PMN infiltration in soft tissues of tooth extraction sockets compared with all other groups (Fig. 7a-c). Interestingly, CY/ZA combination therapies significantly decreased the number and area of blood vessels compared with VC and ZA monotherapies, irrespective of CY dose (Fig. 7d-f). Taken together, in regard to osseous and soft tissue wound healing, the prevalence of BRONJ-like lesions in mice was increased in a CY dosedependent manner when ZA administration was combined with CY.

\section{Discussion}

In the present study, we showed that CY monotherapy delayed osseous wound healing in a dosedependent manner, although this therapy did not affect soft tissue wound healing. Moreover, we demonstrated that CY/ZA combination therapy prevalently induced BRONJ-like lesions in a CY dosedependent manner by inhibiting both osseous and soft tissue wound healing following tooth extraction. 
First, we investigated the effect of CY dose on hard and soft tissue wound healing following tooth extraction. In long bones, $100 \mathrm{mg} / \mathrm{kg}$ of daily CY monotherapy for 7 days negatively affects osteoblasts and osteoclasts, resulting in osteoporosis [22]. It has been demonstrated that $20 \mathrm{mg} / \mathrm{kg}$ of weekly CY injections for more than 5 weeks significantly decreases osteoblast and osteoclast numbers with bone loss in rat mandibular condyles [23]. Another study reported that CY monotherapy for 6 weeks significantly reduced BMD in mice [24]. Decreased BMD induced by CY monotherapy and CY/ZA combination therapy in the current study was in accordance with these previous studies, except we found no osteoclast suppression in tooth extraction sockets. In this study, the CY dose and administration duration were $\geq 50 \mathrm{mg} / \mathrm{kg}$ and 7 weeks, respectively. Moreover, mouse jaw bones instead of mouse long bones or rat mandibular condyles were used in this investigation. Therefore, experimental conditions such as CY dose, administration duration, and experimental species and sites could explain the different effects on osteoclasts on bone surfaces in tooth extraction.

After injury, including tooth extraction, PMNs and macrophages migrate from blood vessels to wounds prevent bacterial infection [25]. Blood vessel formation in wounds is requisite not only for the recruitment of immune cells but also to supply oxygen, nutrition, and numerous cytokines and growth factors [26]. Surprisingly, in this study, soft tissue wound healing normally occurred with respect to collagen production and PMN infiltration, although all doses of CY inhibited angiogenesis. CY has been demonstrated to induce myelosuppression, which induces immunosuppression and decreases hematopoietic stem and progenitor cells [27]. A study in human patients reported that CY chemotherapy induced erythema, ulceration, oral candidiasis, increased plaque accumulation, and severe gingival inflammation [28]. Hence, these studies suggest that CY negatively influences immune responses to oral bacteria. However, recently, it has been demonstrated that high mobility group box 1 proteins enhanced by CY monotherapy activate innate immunity without neutrophil, macrophage, and NK cell suppression in mice [29]. The immune suppression may not be induced by CY monotherapy in this study, even though compromised wound healing of tooth extraction sockets occurred with anti-angiogenesis and no increased PMN infiltration. 
Next, we investigated the effect of ZA on tooth extraction socket healing. As expected, ZA administration suppressed osteoclast numbers. ZA monotherapy did not induce BRONJ-like lesions and resulted in normal collagen production, normal PMN infiltration, and similar angiogenesis to VC injection in the connective tissues of tooth extraction sockets. These findings were in accordance with our previous studies [18, 30], suggesting that our experimental conditions such as ZA dose, injection duration, administration route, and the timing of tooth extraction do not cause the development of BRONJ-like lesions following tooth extraction. Moreover, the International Task Force on ONJ has agreed that antiangiogenesis does not occur when bisphosphonates are used in animal studies [3]. However, other animal studies have reported that ZA monotherapy induces BRONJ-like lesions or compromises wound healing following tooth extraction in rodents [31]. It has also been reported that ZA monotherapy infrequently induces BRONJ following tooth extraction in humans [4]. Hence, triggering factors in addition to tooth extraction may contribute to the development of BRONJ in humans but not in mice. Additional experimental conditions should be tested to clarify the reasons why ZA monotherapy did not induce BRONJ-like lesions in the current study.

Finally, we also investigated the combined effect of CY and ZA. The effect of CY/ZA combination therapy on tooth extraction socket healing significantly differed from that of ZA monotherapy. CY/ZA combination therapies decreased BMD of tooth extraction sockets with increased necrotic bone and empty lacunae. It has been demonstrated that low BMD is highly correlated with the onset of spontaneous osteonecrosis of the knee, although the selected patients did not use bisphosphonates [32]. Low BMD of tooth extraction sockets may be one of the first characteristics of BRONJ-like lesions induced by CY/ZA combination therapy. Over $20 \%$ and $90 \%$ of open soft tissue wounds were observed to have severely reduced collagen fiber production and significant PMN infiltration in CY-M/ZA and CY-H/ZA groups, respectively. As a result, CY/ZA combination therapies severely influenced the soft and hard tissues of extraction sockets, resulting in frequent induction of BRONJ-like lesions in a CY dose-dependent manner. Interestingly, histological data showed that the degree of angiogenesis inhibition following CY/ZA combination therapies was almost the same as that of CY monotherapy, although all doses of CY 
monotherapies and the CY-L/ZA combination therapy rarely induced BRONJ-like lesions. Thus, in addition to an anti-angiogenesis effect on tooth extraction socket healing, other factors exacerbating tooth extraction socket healing may exist only after CY/ZA combination therapy is performed in mice. Caution should be taken in dental treatment, including tooth extraction, when high doses of chemotherapeutic agents are used with bisphosphonates in clinical situations.

\section{Conclusions}

In the present study, we demonstrated that CY therapy alone did not induce open wounds in mice, irrespective of CY dose, although CY monotherapy delayed osseous healing in a CY dosedependent manner. ZA monotherapy did not induce BRONJ-like lesions. Moreover, we showed that BRONJ-like lesions were prevalently induced in a CY dose-dependent manner when tooth extraction was performed in mice treated with CY/ZA combination therapy.

\section{Acknowledgements}

This work was supported by JSPS KAKENHI Grant Numbers 25870523, 15K11258 and $18 \mathrm{H} 02994$.

\section{Conflict of Interest}

The authors declare that they have no conflicts of interest

\section{References}

[1] J.J. Body, R. Bartl, P. Burckhardt, P.D. Delmas, I.J. Diel, H. Fleisch, J.A. Kanis, R.A. Kyle, G.R. Mundy, A.H. Paterson, R.D. Rubens, Current use of bisphosphonates in oncology. International Bone and Cancer Study Group, J Clin Oncol 16 (1998) 3890-3899. https://doi.org/10.1200/JCO.1998.16.12.3890 
[2] R.E. Marx, Pamidronate (Aredia) and zoledronate (Zometa) induced avascular necrosis of the jaws: a growing epidemic, J Oral Maxillofac Surg. 61 (2003) 1115-1117, https://doi.org/10.1016/S0278$\underline{2391(03) 00720-1}$

[3] A.A. Khan, A. Morrison, D.A. Hanley, D. Felsenberg, L.K. McCauley, F. O'Ryan, I.R. Reid, S.L. Ruggiero, A. Taguchi, S. Tetradis, N.B. Watts, M.L. Brandi, E. Peters, T. Guise, R. Eastell, A.M. Cheung, S.N. Morin, B. Masri, C. Cooper, S.L. Morgan, B. Obermayer-Pietsch, B.L. Langdahl, R. Al Dabagh, K.S. Davison, D.L. Kendler, G.K. Sándor, R.G. Josse, M. Bhandari, M. El Rabbany, D.D. Pierroz, R. Sulimani, D.P. Saunders, J.P. Brown, J. Compston, I.T.F.o.O.o.t. Jaw, Diagnosis and management of osteonecrosis of the jaw: a systematic review and international consensus, J Bone Miner Res. 30 (2015) 3-23, https://doi.org/10.1002/jbmr.2405

[4] S.L. Ruggiero, T.B. Dodson, J. Fantasia, R. Goodday, T. Aghaloo, B. Mehrotra, F. O'Ryan, A.A.o.O.a.M. Surgeons, American Association of Oral and Maxillofacial Surgeons position paper on medication-related osteonecrosis of the jaw--2014 update, J Oral Maxillofac Surg. 72 (2014) 1938-1956, https://doi.org/10.1016/i.joms.2014.04.031

[5] R. Fliefel, M. Tröltzsch, J. Kühnisch, M. Ehrenfeld, S. Otto, Treatment strategies and outcomes of bisphosphonate-related osteonecrosis of the jaw (BRONJ) with characterization of patients: a systematic review, Int J Oral Maxillofac Surg 44 (2015) 568-585. https://doi.org/10.1016/i.ijom.2015.01.026

[6] R.A. Miksad, K.C. Lai, T.B. Dodson, S.B. Woo, N.S. Treister, O. Akinyemi, M. Bihrle, G. Maytal, M. August, G.S. Gazelle, J.S. Swan, Quality of life implications of bisphosphonate-associated osteonecrosis of the jaw, Oncologist 16 (2011) 121-132, https://doi.org/10.1634/theoncologist.2010-0183

[7] D. Pulte, L. Jansen, F.A. Castro, K. Emrich, A. Katalinic, B. Holleczek, H. Brenner, G.C.S.W. Group, Trends in survival of multiple myeloma patients in Germany and the United States in the first decade of the 21st century, Br J Haematol. 171 (2015) 189-196, https://doi.org/10.1111/bjh.13537

[8] S.V. Rajkumar, S. Kumar, Multiple Myeloma: Diagnosis and Treatment, Mayo Clin Proc 91(1) (2016) 101-119. https://doi.org/10.1016/j.mayocp.2015.11.007 
[9] C. Röllig, S. Knop, M. Bornhäuser, Multiple myeloma, Lancet 385 (2015) 2197-2208, https://doi.org/10.1016/S0140-6736(14)60493-1

[10] E. Terpos, I.M. Society, Multiple Myeloma: Clinical Updates From the American Society of Hematology Annual Meeting, 2017, Clin Lymphoma Myeloma Leuk (2018). https://doi.org/10.1016/i.clml.2018.02.015

[11] M. Sasaki, S. Kuroshima, Y. Aoki, N. Inaba, T. Sawase, Ultrastructural alterations of osteocyte morphology via loaded implants in rabbit tibiae, J Biomech. 48 (2015) 4130-4141, https://doi.org/10.1016/i.jbiomech.2015.10.025

[12] S. Kuroshima, B.L. Kovacic, K.M. Kozloff, L.K. McCauley, J. Yamashita, Intra-oral PTH administration promotes tooth extraction socket healing, J Dent Res. 92 (2013) 553-559, https://doi.org/10.1177/0022034513487558

[13] M.L. Bouxsein, S.K. Boyd, B.A. Christiansen, R.E. Guldberg, K.J. Jepsen, R. Müller, Guidelines for assessment of bone microstructure in rodents using micro-computed tomography, J Bone Miner Res. 25 (2010) 1468-1486, https://doi.org/10.1002/jbmr.141

[14] A.M. Parfitt, Z.H. Han, S. Palnitkar, D.S. Rao, M.S. Shih, D. Nelson, Effects of ethnicity and age or menopause on osteoblast function, bone mineralization, and osteoid accumulation in iliac bone, J Bone Miner Res. 12 (1997) 1864-1873, https://doi.org/10.1359/jbmr.1997.12.11.1864

[15] R.S. Weinstein, R.L. Jilka, A.M. Parfitt, S.C. Manolagas, Inhibition of osteoblastogenesis and promotion of apoptosis of osteoblasts and osteocytes by glucocorticoids. Potential mechanisms of their deleterious effects on bone, J Clin Invest. 102 (1998) 274-282, https://doi.org/10.1172/JCl2799

[16] C. Fondi, A. Franchi, Definition of bone necrosis by the pathologist, Clin Cases Miner Bone Metab. 4 (2007) 21-26.

[17] Q. Zhang, I. Atsuta, S. Liu, C. Chen, S. Shi, A.D. Le, IL-17-mediated M1/M2 macrophage alteration contributes to pathogenesis of bisphosphonate-related osteonecrosis of the jaws, Clin Cancer Res. 19 (2013) 3176-3188, https://doi.org/10.1158/1078-0432.CCR-13-0042 
[18] S. Kuroshima, J. Yamashita, Chemotherapeutic and antiresorptive combination therapy suppressed lymphangiogenesis and induced osteonecrosis of the jaw-like lesions in mice, Bone 56 (2013) 101-109, https://doi.org/10.1016/j.bone.2013.05.013

[19] J. Yamashita, K. Koi, D.Y. Yang, L.K. McCauley, Effect of zoledronate on oral wound healing in rats, Clin Cancer Res. 17 (2011) 1405-1414, https://doi.org/10.1158/1078-0432.CCR-10-1614

[20] F.C. Barone, L.M. Hillegass, W.J. Price, R.F. White, E.V. Lee, G.Z. Feuerstein, H.M. Sarau, R.K. Clark, D.E. Griswold, Polymorphonuclear leukocyte infiltration into cerebral focal ischemic tissue: myeloperoxidase activity assay and histologic verification, J Neurosci Res 29 (1991) 336-45. https://doi.org/10.1002/jnr.490290309

[21] Y. Akita, S. Kuroshima, K. Nakajima, H. Hayano, R. Kanai, M. Sasaki, T. Sawase, Effect of antiangiogenesis induced by chemotherapeutic monotherapy, chemotherapeutic/bisphosphonate combination therapy and anti-VEGFA mAb therapy on tooth extraction socket healing in mice, J Bone Miner Metab. (2017), https://doi.org/10.1007/s00774-017-0872-1

[22] D. Zhao, C. Wang, Y. Zhao, B. Shu, Y. Jia, S. Liu, H. Wang, J. Chang, W. Dai, S. Lu, Q. Shi, Y. Yang, Y. Zhang, Y. Wang, Cyclophosphamide causes osteoporosis in C57BL/6 male mice: suppressive effects of cyclophosphamide on osteoblastogenesis and osteoclastogenesis, Oncotarget 8(58) (2017) 9816398183, https://doi.org/10.18632/oncotarget.21000

[23] T.M. Wang, C. Shih, Study of histomorphometric changes of the mandibular condyles in neonatal and juvenile rats after administration of cyclophosphamide, Acta Anat. (Basel) 127 (1986) 93-99,

[24] T. Ponnapakkam, R. Katikaneni, T. Nichols, G. Tobin, J. Sakon, O. Matsushita, R.C. Gensure, Prevention of chemotherapy-induced osteoporosis by cyclophosphamide with a long-acting form of parathyroid hormone, J Endocrinol Invest. 34 (2011) e392-e397, https://doi.org/10.3275/7864

[25] S.A. Eming, T. Krieg, J.M. Davidson, Inflammation in wound repair: molecular and cellular mechanisms, J Invest Dermatol. 127 (2007) 514-525, https://doi.org/10.1038/si.jid.5700701

[26] A.E. Vieira, C.E. Repeke, S.e.B. Ferreira Junior, P.M. Colavite, C.C. Biguetti, R.C. Oliveira, G.F. Assis, R. Taga, A.P. Trombone, G.P. Garlet, Intramembranous bone healing process subsequent to tooth 
extraction in mice: micro-computed tomography, histomorphometric and molecular characterization, PLoS One 10 (2015) e0128021. https://doi.org/10.1371/journal.pone.0128021

[27] L. Feng, Q. Huang, Z. Huang, H. Li, X. Qi, Y. Wang, Z. Liu, X. Liu, L. Lu, Optimized Animal Model of Cyclophosphamide-induced Bone Marrow Suppression, Basic Clin Pharmacol Toxicol 119 (2016) 428435. https://doi.org/10.1111/bcpt.12600

[28] S.B. Jensen, H.T. Mouridsen, O.J. Bergmann, J. Reibel, N. Brünner, B. Nauntofte, Oral mucosal lesions, microbial changes, and taste disturbances induced by adjuvant chemotherapy in breast cancer patients, Oral Surg Oral Med Oral Pathol Oral Radiol Endod. 106 (2008) 217-226, https://doi.org/10.1016/j.tripleo.2008.04.003

[29] J.L. Guerriero, D. Ditsworth, J.M. Catanzaro, G. Sabino, M.B. Furie, R.R. Kew, H.C. Crawford, W.X. Zong, DNA alkylating therapy induces tumor regression through an HMGB1-mediated activation of innate immunity, J Immunol. 186 (2011) 3517-3526, https://doi.org/10.4049/immunol.1003267

[30] S. Kuroshima, M. Sasaki, K. Nakajima, S. Tamaki, H. Hayano, T. Sawase, Transplantation of Noncultured Stromal Vascular Fraction Cells of Adipose Tissue Ameliorates Osteonecrosis of the JawLike Lesions in Mice, J Bone Miner Res 33 (2018) 154-166, https://doi.org/10.1002/jbmr.3292

[31] Y. Bi, Y. Gao, D. Ehirchiou, C. Cao, T. Kikuiri, A. Le, S. Shi, L. Zhang, Bisphosphonates cause osteonecrosis of the jaw-like disease in mice, Am J Pathol. 177 (2010) 280-290, https://doi.org/10.2353/ajpath.2010.090592

[32] Y. Akamatsu, N. Mitsugi, T. Hayashi, H. Kobayashi, T. Saito, Low bone mineral density is associated with the onset of spontaneous osteonecrosis of the knee, Acta Orthop. 83 (2012) 249-255, https://doi.org/10.3109/17453674.2012.684139 


\section{Figure legends}

Fig. 1. Experimental design. Time schedules for ZA monotherapy, CY monotherapy, and CY/ZA combination therapy. Tooth extraction was performed at 3 weeks after the onset of drug treatments. Euthanasia was conducted at 4 weeks after tooth extraction. VC: vehicle control, CY: cyclophosphamide, ZA: zoledronate.

Fig. 2. Effect of CY monotherapy on bone healing in tooth extraction sockets. (a) Representative intraoral views (M2: second molar, M3: third molar, red arrow: open wound). (b) Representative sagittal H-E- and TRAP-stained images of tooth extraction sockets (Bar: $100 \mu \mathrm{m}$ and $50 \mu \mathrm{m}$, respectively). Dotted lines in H-E-stained images indicate extraction sockets. No necrotic bone and wound open areas were noted. Black arrowheads in TRAP-stained images indicate osteoclasts on bone surfaces. (c) Osteoclast number (N.Oc/BS) remained unchanged, irrespective of $C Y$ dose. (d) Living bone area was significantly decreased in CY-M and CY-H vs. VC. (e) Necrotic bone area was significantly increased in CY-M and CY-H vs. VC. (f) The number of empty lacunae was significantly increased in CY-M and CY-H vs. VC in a dose-dependent manner. (g) The number of osteocytes was significantly decreased in $\mathrm{CY}-\mathrm{H}$ vs. VC and CY-L. $\mathrm{n}=7$ /group; ${ }^{*}, p<0.05,{ }^{* *}, p<0.01,{ }^{* * *}, p<0.001$, compared with VC. ${ }^{\dagger}, p<.05,{ }^{\dagger \dagger}, p<.01$, compared with groups except for VC.

Fig. 3. Effect of CY monotherapy on bone architecture of tooth extraction sockets. (a) Representative cross-sectional microCT images of tooth extraction sockets. M1 and M2 indicate first molars and second molars, respectively. (b) Bone fill was significantly smaller in CY-H vs. VC, CY-L, and CY-M. (c) Trabecular number (Tb.N) was significantly decreased in CY-M and CY-H vs. VC. (d) Trabecular thickness (Tb.Th) was the same in all groups. (e) Trabecular separation (Tb.Sp) was significantly increased in CY-H vs. all other groups. (f) Trabecular bone mineral density (Tb.BMD) was significantly 
decreased in CY-L, CY-M, and CY-H vs. VC. $\mathrm{n}=7$ /group; ${ }^{*}, p<0.05,{ }^{* * *}, p<0.001$, compared with VC. ${ }^{\dagger}, p<.05,{ }^{\dagger+}, p<.001$, compared with groups except for VC.

Fig. 4. Effect of CY monotherapy on soft tissue healing in tooth extraction sockets. (a) Representative sagittal images of trichrome staining in tooth extraction sockets (Bar: $200 \mu \mathrm{m}$ ). Yellow dotted lines indicate the border between alveolar bone and connective tissue.). (b) Representative sagittal images of CD31 immunostaining. Nuclei were stained with DAPI (Bar: $100 \mu \mathrm{m})$. White arrowheads indicate CD31-positive vessels. (c) Collagen area was nearly the same among groups, irrespective of CY. (d) The number of inflammatory cells was the same among groups. (e) The number of blood vessels was significantly decreased in CY-L, CY-M, and CY-H vs. VC. (f) Vessel area was significantly smaller in CY-L, CY-M, and CY-H vs. VC. $\mathrm{n}=7 /$ group; ${ }^{*}, p<0.05,{ }^{* *}, p<0.01,{ }^{* * *}, p<0.001$, compared with VC.

Fig. 5. Effect of ZA monotherapy and CY/ZA combination therapy on osseous healing of tooth extraction sockets. (a) Representative intraoral views. (M2: second molar, M3: third molar, red arrow: open wound). (b) Representative sagittal H-E- and TRAP-stained images of tooth extraction sockets (Bar: $100 \mu \mathrm{m}$ and $50 \mu \mathrm{m}$, respectively). Dotted lines in $\mathrm{H}$-E-stained images indicate extraction sockets. Black arrowheads in TRAP-stained images indicate osteoclasts on bone surfaces. (c) ZA therapy significantly decreased osteoclast number (N.Oc/BS) compared with VC, regardless of CY administration. (d) Living bone area was the same between VC and ZA monotherapy, whereas living bone area was significantly decreased in CY-L/ZA, CY-M/ZA, and CY-H/ZA vs. VC. (e) Necrotic bone area was greater in ZA vs. VC, although the difference was not significant. Necrotic bone area was significantly increased in CY-L/ZA, CY-M/ZA, and $\mathrm{CY}-\mathrm{H} / \mathrm{ZA}$ vs. VC and ZA. The greatest necrotic bone area was noted in CY-H/ZA. (f) The number of empty lacunae was greater in ZA vs. VC, although the difference was not significant. The number of empty lacunae in tooth extraction sockets was significantly increased in CY-L/ZA, CY-M/ZA, and CY- 
H/ZA vs. VC and ZA. (g) ZA therapy significantly decreased the number of osteocytes compared with that in $\mathrm{VC}$, regardless of $\mathrm{CY}$ administration. $\mathrm{n}=7 /$ group; ${ }^{*}, p<0.05,{ }^{* *}, p<0.01,{ }^{* * *}, p<0.001$, compared with VC. ${ }^{\dagger \dagger}, p<.01,{ }^{\dagger \dagger}, p<.001$, compared with groups except for VC.

Fig. 6. Effect of ZA monotherapy and CY/ZA combination therapy on bone structures in tooth extraction sockets. (a) Representative cross-sectional microCT images of tooth extraction sockets. M1 and M2 indicate first and second molars, respectively. (b) Bone fill of tooth extraction sockets was significantly impaired in CY-H/ZA vs. all other groups. Bone fill in tooth extraction sockets was significantly higher in ZA vs VC. (c) Trabecular number (Tb.N) was significantly decreased in ZA, CY-M/ZA, and CY-H/ZA vs. VC. (d) Trabecular thickness (Tb.Th) was significantly smaller in CY-H/ZA vs. all other groups. (e) Trabecular separation (Tb.Sp) was significantly greater in CY-H/ZA vs. all other groups. (f) Bone mineral density (BMD) was significantly increased in ZA vs. VC. BMD was significantly decreased in CY/ZA vs. VC and ZA, irrespective of CY- dose. $\mathrm{n}=7 /$ group; ${ }^{*}, p<0.05,{ }^{* * *}, p<0.001$, compared with VC. ${ }^{\mathrm{ttt}}, p<$ 0.001, compared with groups except for VC.

Fig. 7. Effect of ZA monotherapy and CY/ZA combination therapy on soft tissue wound healing of tooth extraction sockets. (a) Representative trichrome-stained images of tooth extraction sockets (Bar: $200 \mu \mathrm{m}$. Yellow dotted lines indicate the border between alveolar bone and connective tissue.). (b) Representative immunostaining for CD31. Nuclei were stained using DAPI (Bar: $100 \mu \mathrm{m})$. White arrowheads indicate CD31-positive vessels. (c) Collagen area was significantly decreased in CY-H/ZA vs. all other groups. (d) A significantly greater number of infiltrated inflammatory cells were observed in CY-H/ZA vs. all other groups. (e) The number of blood vessels was the same between VC and ZA. The vessel number was significantly decreased in CY-L/ZA, CY-M/ZA, and CY-H/ZA vs. VC and ZA. (f) Vessel area was the same between VC and ZA. Vessel area was significantly decreased in CY-L/ZA, CY-M/ZA, and CY-H/ZA vs. 
VC and ZA. $\mathrm{n}=7$ /group; ${ }^{* *}, p<0.001$, compared with VC. ${ }^{\dagger}, p<0.05,{ }^{\dagger \dagger}, p<0.01,{ }^{t+\dagger}, p<0.001$ compared with groups except for VC.

Fig. 1.

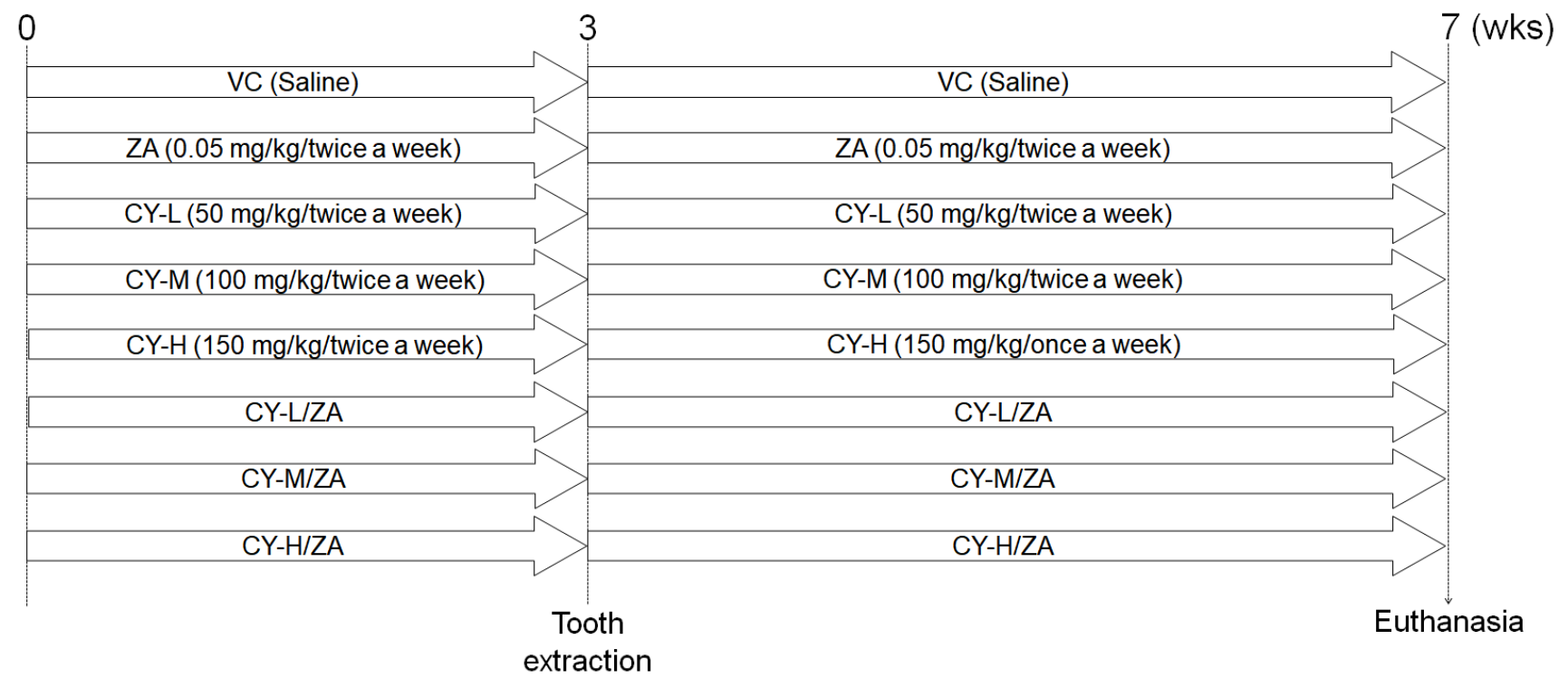


Fig. 2.

(a)
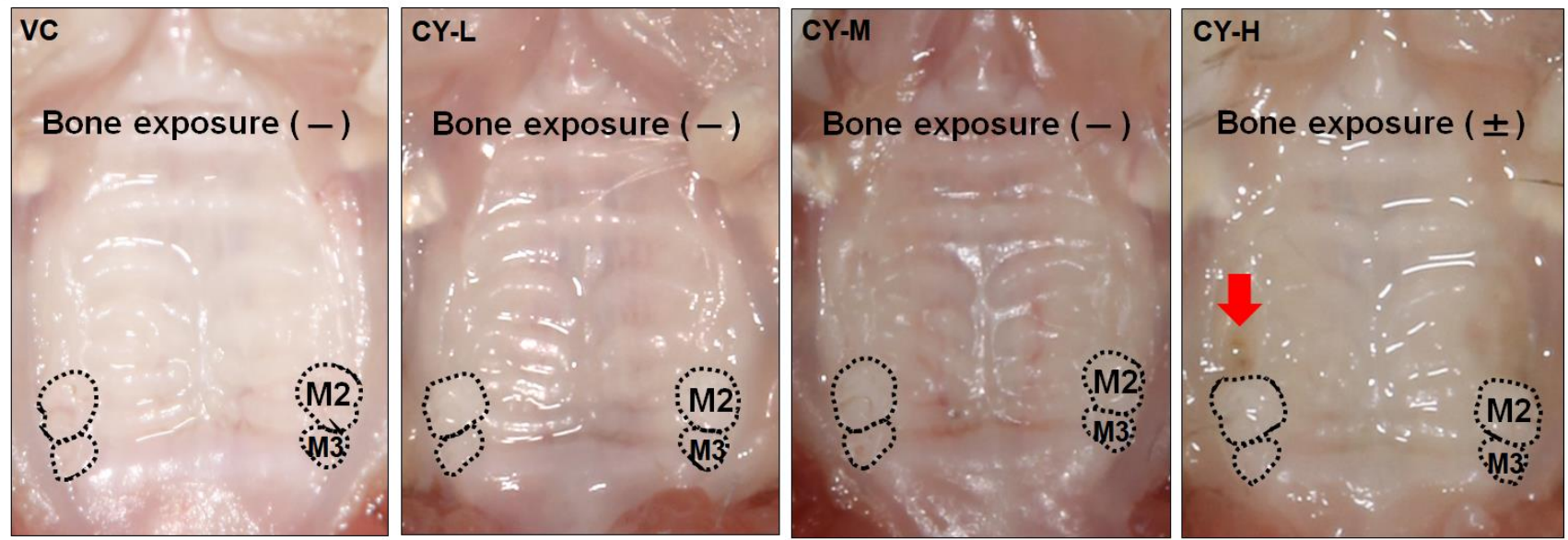

(b)
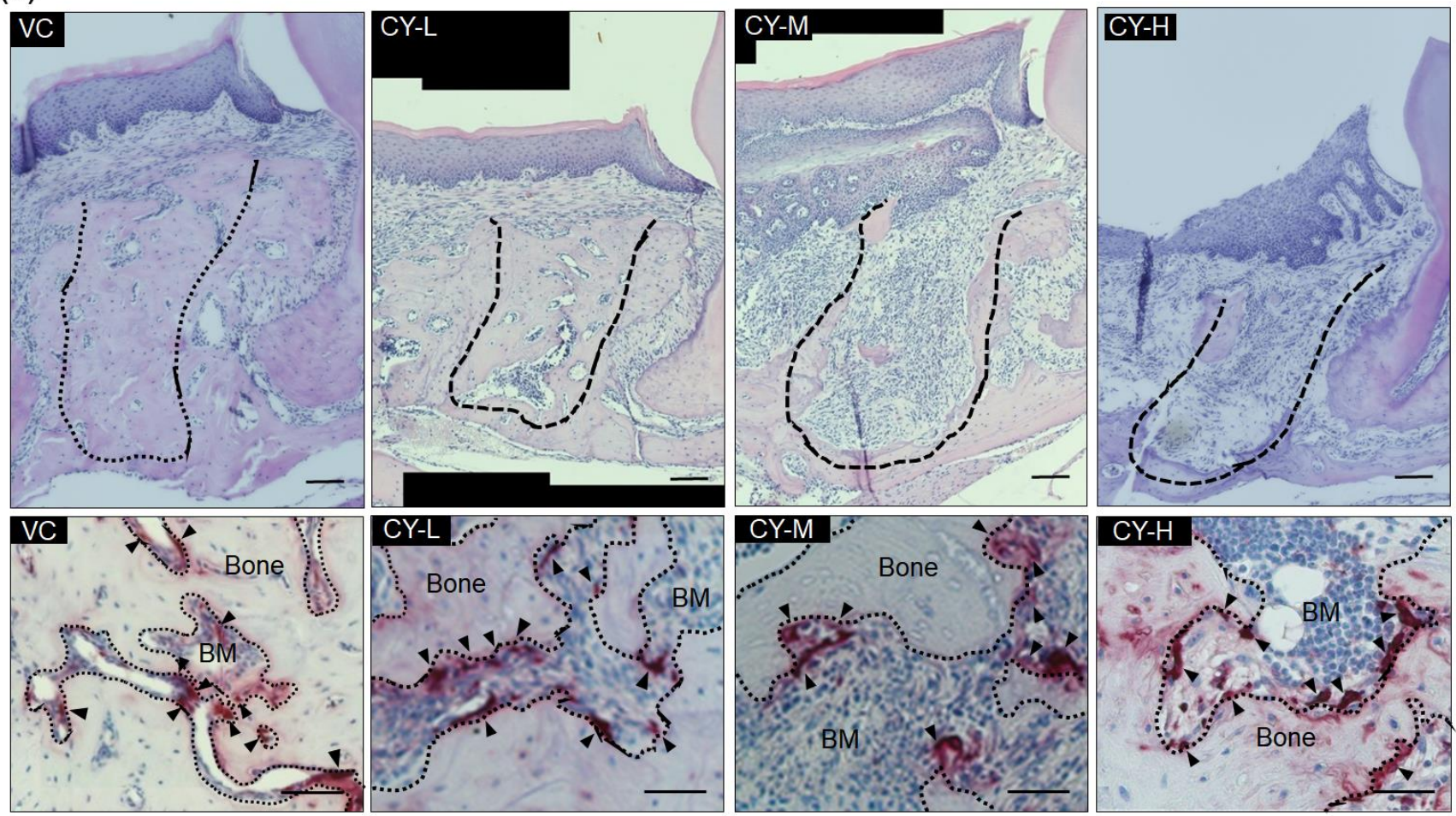

(c)

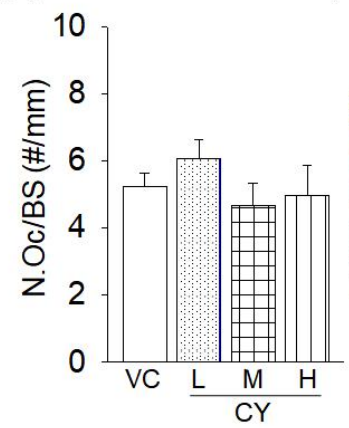

(d)

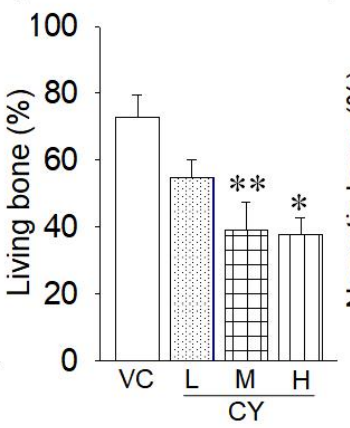

(e)

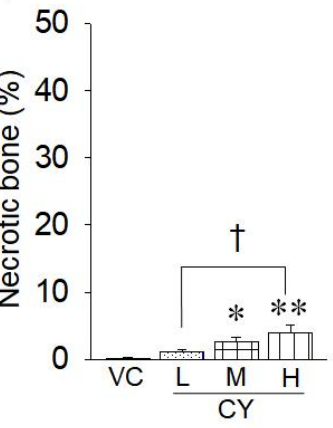

(f)

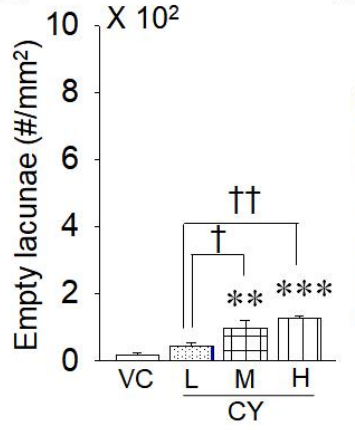

(g)

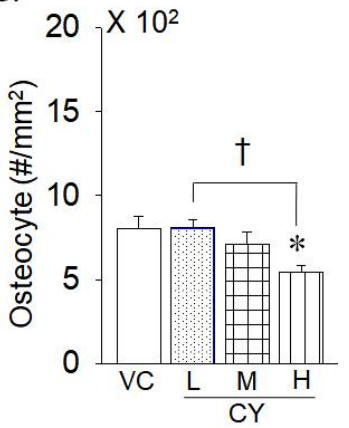


Fig. 3.

(a)

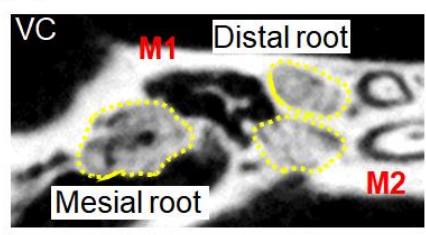

(b)

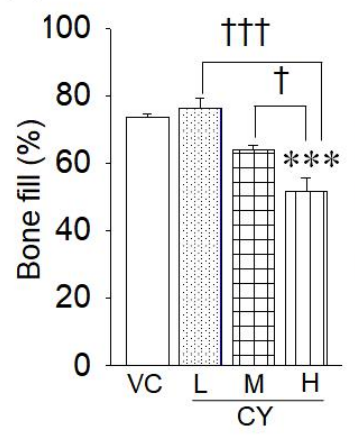

(c)

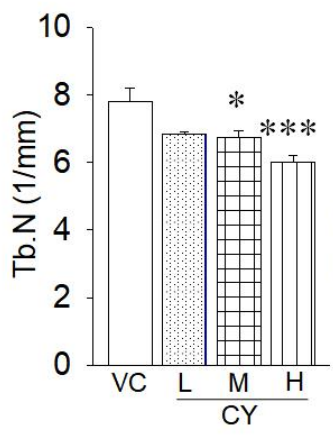

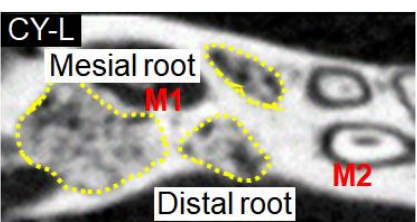

Distal root

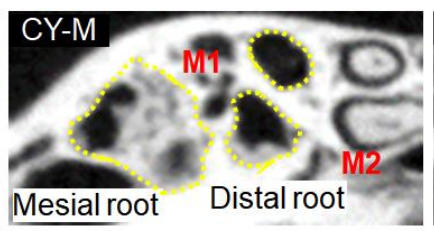

(e)

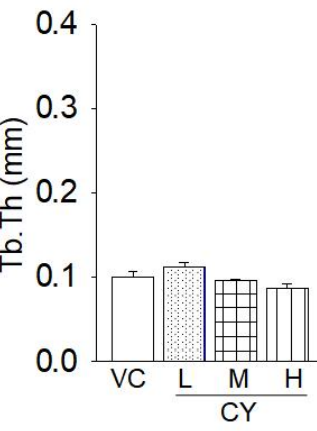

CY-H

Mesial root

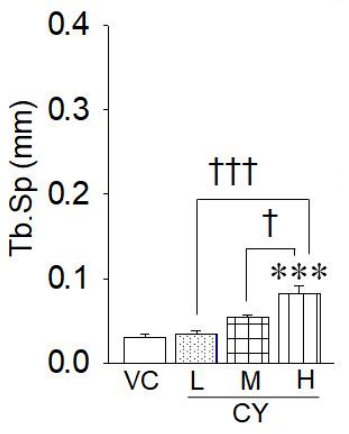

(f)

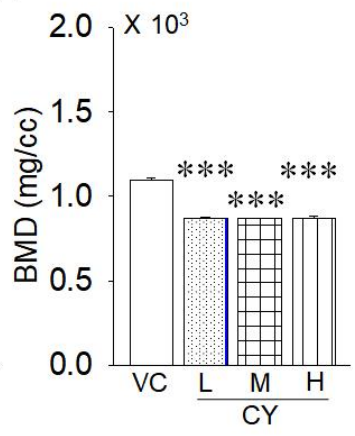


Fig. 4.

(a)
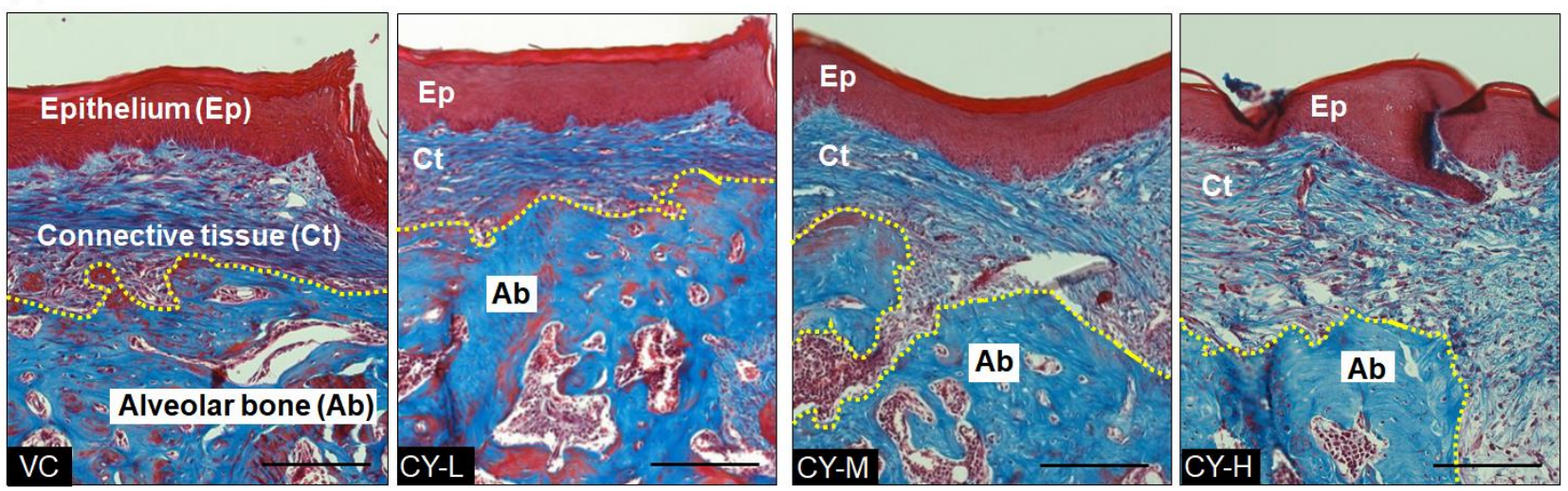

(b)

(c)
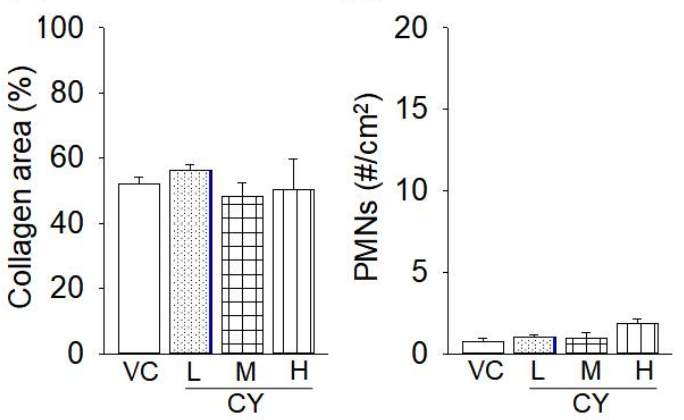

(d)
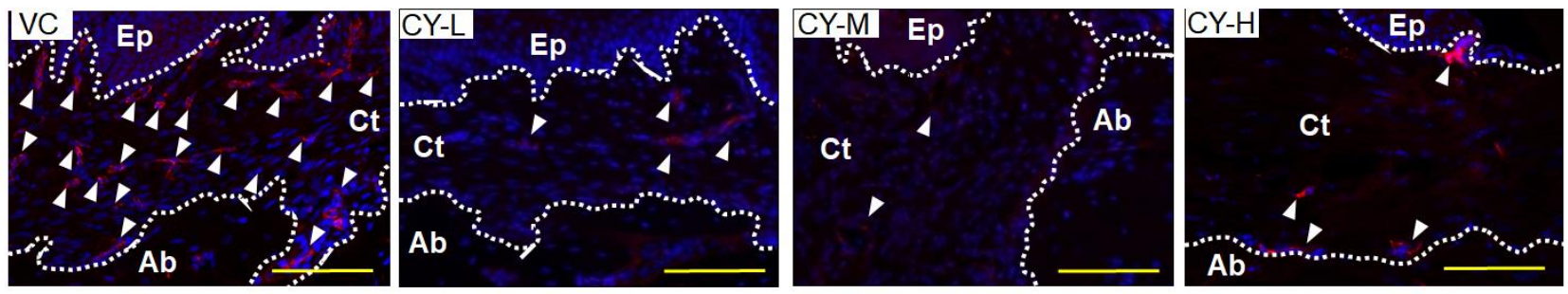

(e)

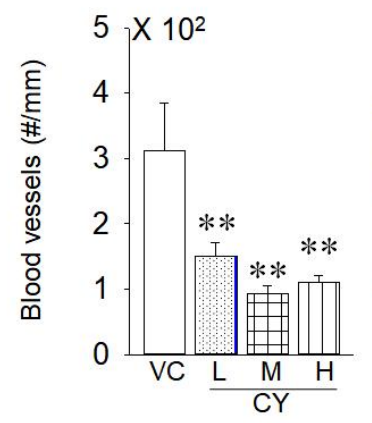

(f)

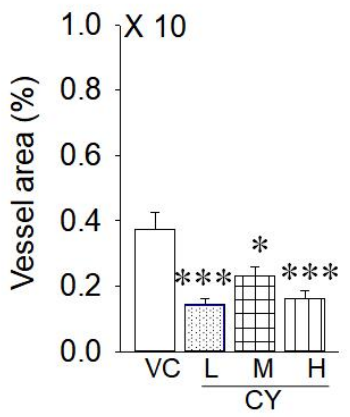


Fig. 5.

(a)
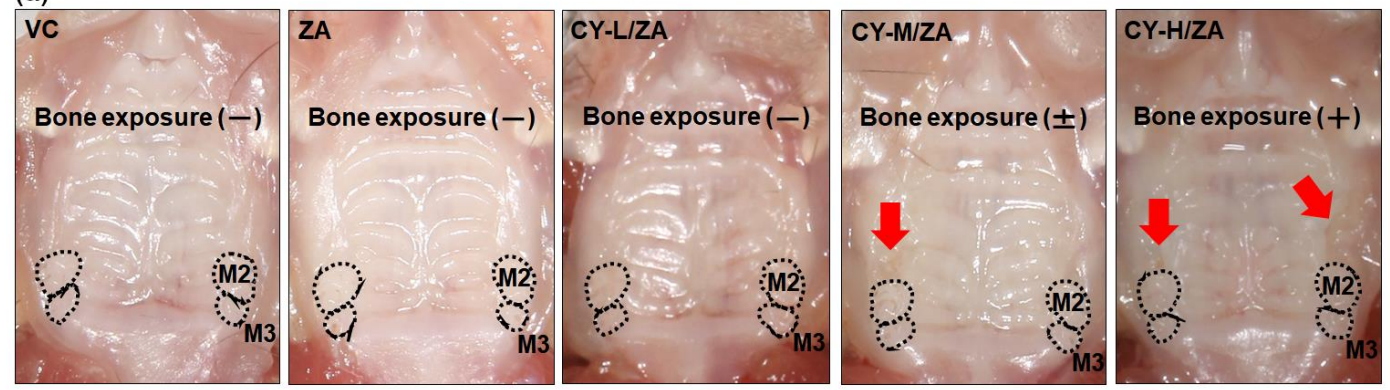

(b)
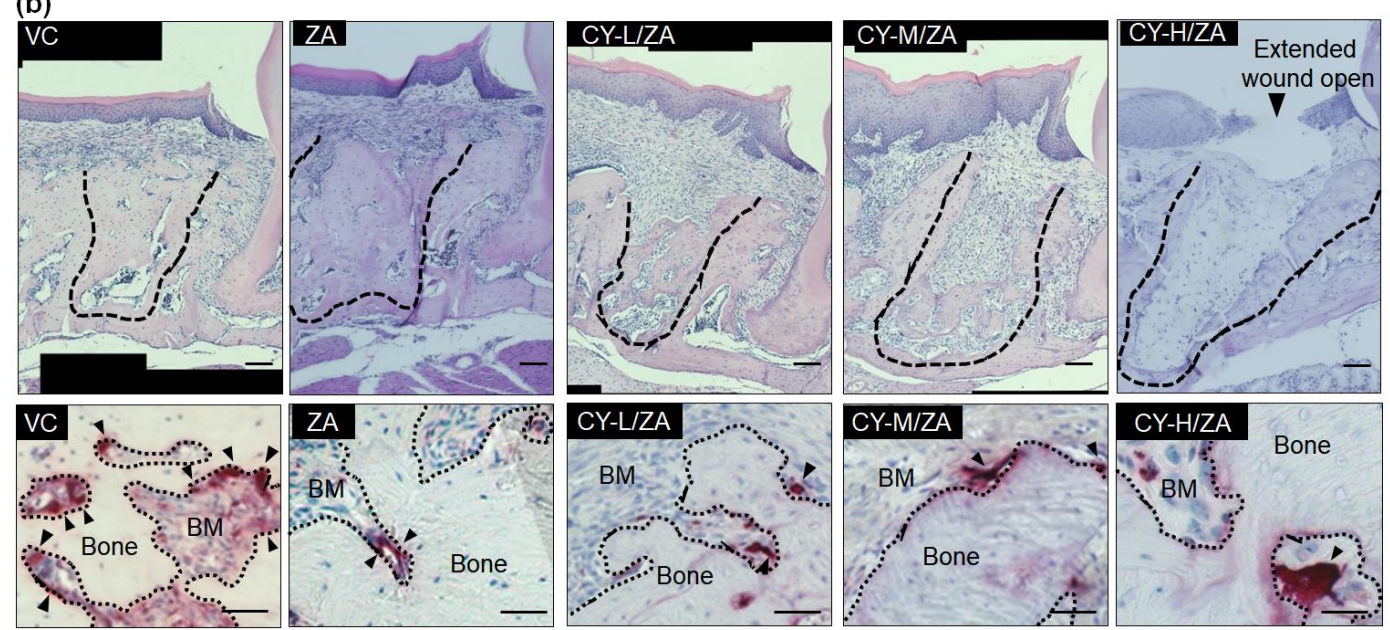

(c)

(d)

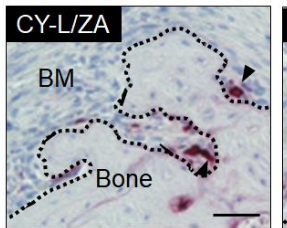

(e)
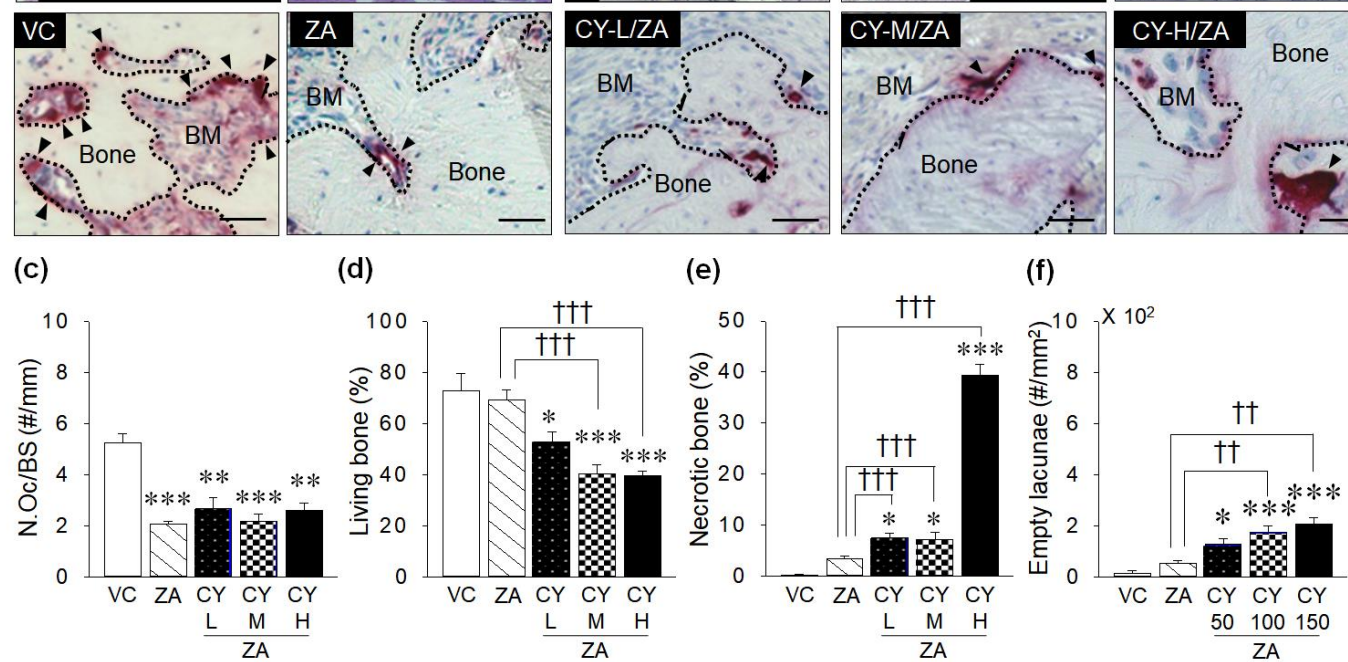

(f)

(g)

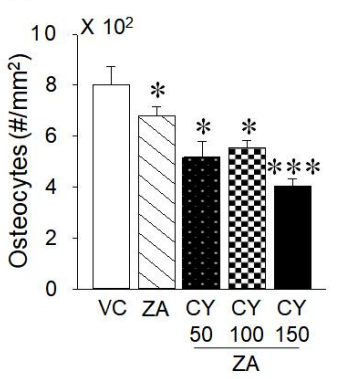


Fig. 6 .

(a)
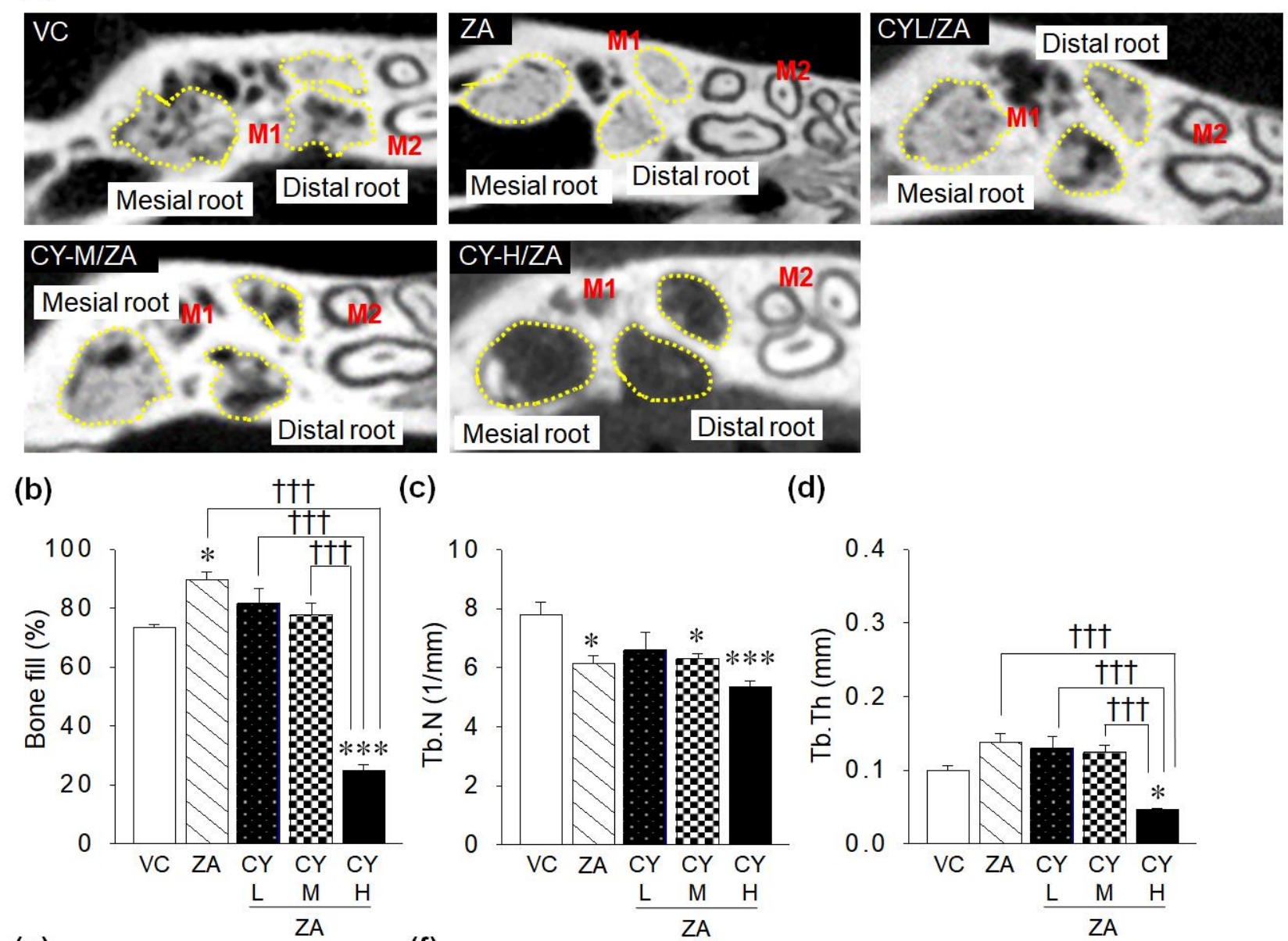

(c)

(d)

(e)

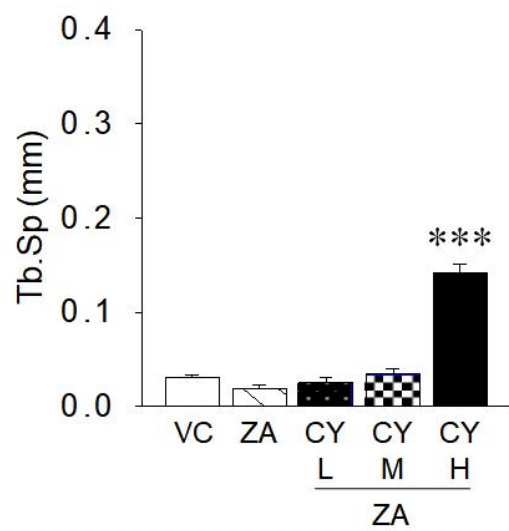

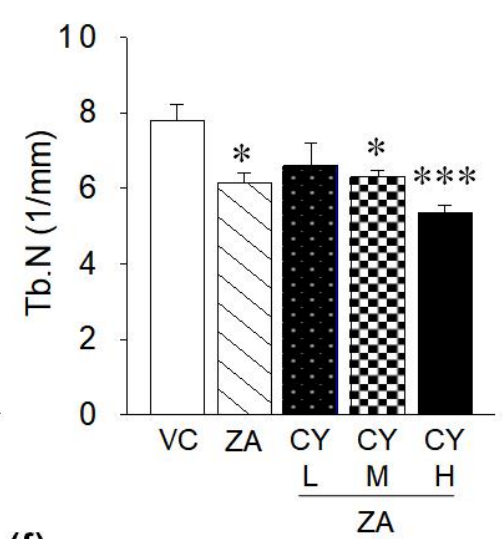

(f)

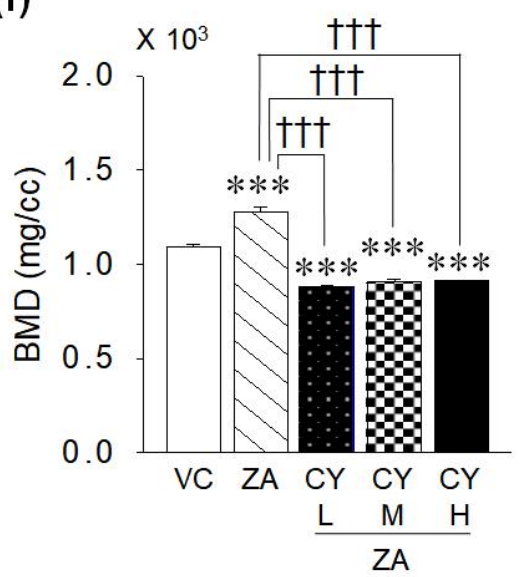

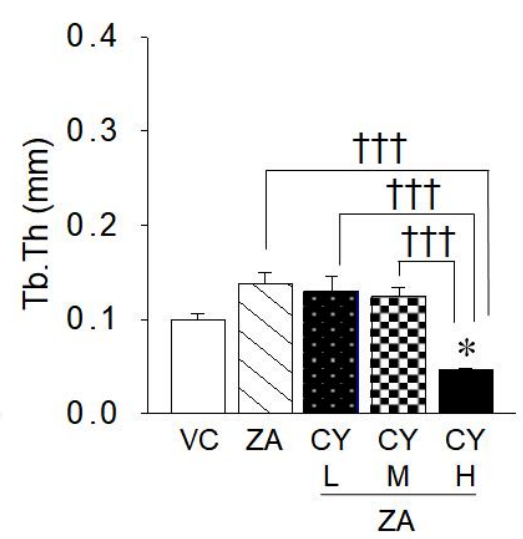


Fig. 7 .

(a)

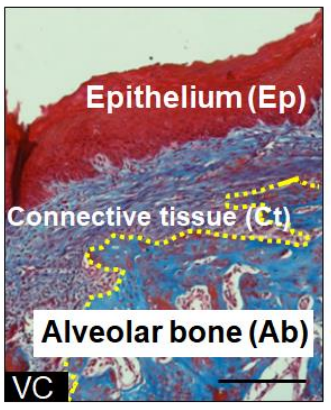

(b)

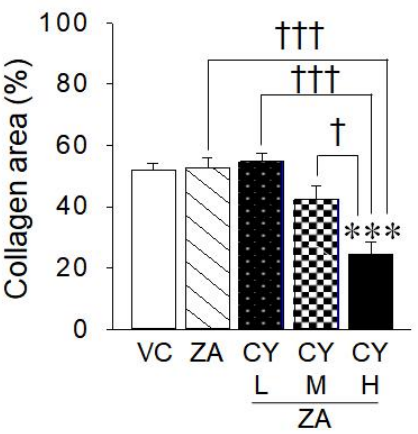

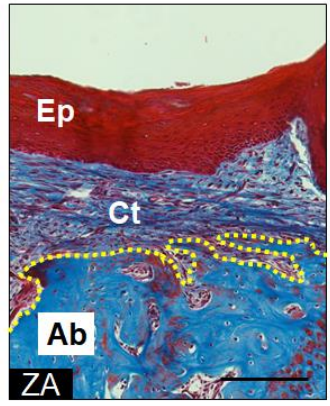
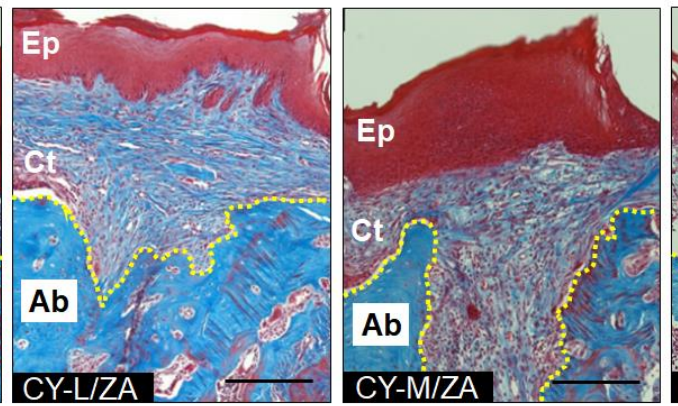

(c)

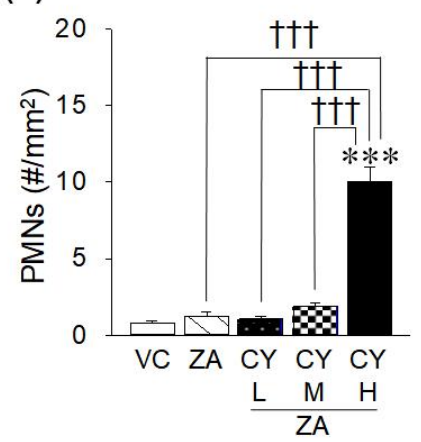

(d)

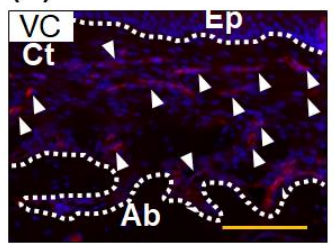

(e)

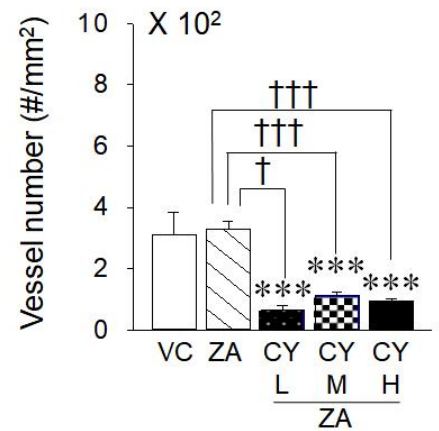

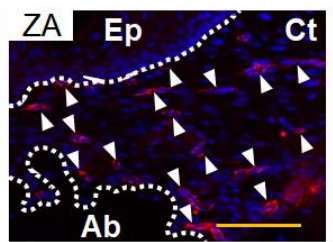

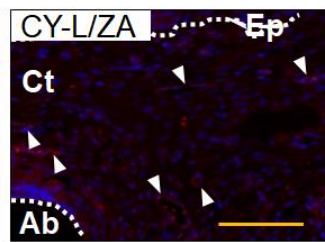

(f)

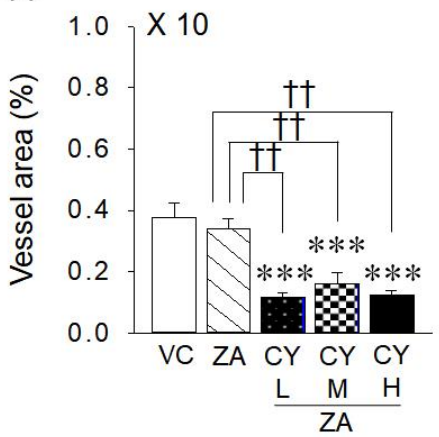

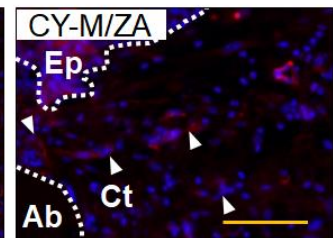

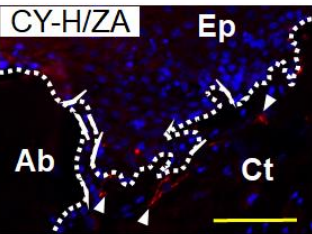

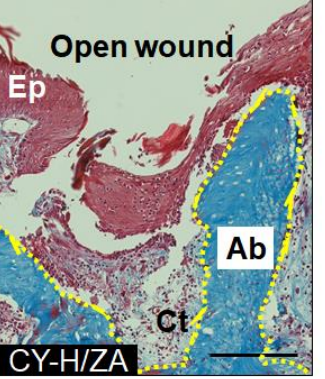

